\title{
Charge Transfer in Model Peptides: Obtaining Marcus Parameters from Molecular Simulation
}

\author{
Alexander Heck, ${ }^{\dagger}$ P. Benjamin Woiczikowski, ${ }^{\dagger}$ Tomáš Kubař, $^{\dagger}$ Bernd Giese, $^{\ddagger}$ Marcus Elstner, ${ }^{\dagger}$ \\ and Thomas B. Steinbrecher*, ${ }^{*}$ \\ †Department for Theoretical Chemical Biology, Institute for Physical Chemistry, Karlsruhe Institute of Technology, Kaiserstr. 12, \\ 76131 Karlsruhe, Germany \\ ${ }^{\ddagger}$ Department of Chemistry, University of Fribourg, Chemin du Musee 9, CH-1700 Fribourg, Switzerland
}

\begin{abstract}
Charge transfer within and between biomolecules remains a highly active field of biophysics. Due to the complexities of real systems, model compounds are a useful alternative to study the mechanistic fundamentals of charge transfer. In recent years, such model experiments have been underpinned by molecular simulation methods as well. In this work, we study electron hole transfer in helical model peptides by means of molecular dynamics simulations. A theoretical framework to extract Marcus parameters of charge transfer from simulations is presented. We find that the

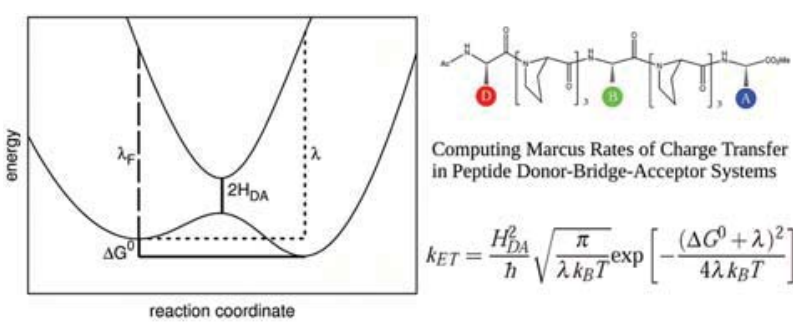
peptides form stable helical structures with sequence dependent small deviations from ideal PPII helices. We identify direct exposure of charged side chains to solvent as a cause of high reorganization energies, significantly larger than typical for electron transfer in proteins. This, together with small direct couplings, makes long-range superexchange electron transport in this system very slow. In good agreement with experiment, direct transfer between the terminal amino acid side chains can be dicounted in favor of a two-step hopping process if appropriate bridging groups exist.
\end{abstract}

\section{INTRODUCTION}

Charge transfer is a fundamental phenomenon in physical chemistry, enjoying strong and consistent scientific interest for more than fifty years. ${ }^{1}$ Understanding charge transfer in complex and flexible biomolecules is crucial for a huge variety of processes, from cellular respiration and photosynthesis to DNA damage and repair. ${ }^{2-4}$ Describing it accurately has turned out to be particularly challenging, both to experimentalists and theoreticians. Biochemical charge transfer involves the directed movement of electrons over large distances (multiple nanometers) through macromolecules, typically proteins or large heterogeneous multiprotein assemblies. These processes involve dynamical changes that occur on a time scale spanning multiple orders of magnitude, from subpicosecond changes in electronic structure to microsecond or even slower conformational changes. Only recently has the complicated interplay between atomic structural fluctuations and electron dynamics become a focus for charge transfer studies in biochemical systems, ${ }^{5-9}$ building onto earlier work on model systems. ${ }^{10-15}$ This fact, combined with the large system dimensions and difficult experimental conditions, explains why many open questions on electron transfer (ET) in biomolecules remain. Therefore, simpler model systems to study some aspects of ET have gained popularity, and theoretical models based on computer simulations have become important tools to help interpret experiments and gain a better understanding of the structure and function of the involved molecules. ${ }^{16-22}$

An important model system in the study of charge transfer processes has always been peptide systems. Extensive experimental work was done by Isied et al., who have studied ET over distances of 8-32 Å via spectroscopical techniques and determined the properties of polyproline II helices as ET bridges. ${ }^{27-34}$ This work has inspired theoretical investigations of peptide charge transfer systems, which suceeded in computing Marcus parameters in good agreement to experiment, ${ }^{35-37}$ an aproach that we will extend to a different peptide system here.

Recently, the group of B. Giese developed a peptide system (Figure 1) in which intramolecular ET takes place between a Cterminal dialkoxy phenylalanine radical cation as electron acceptor, and an $\mathrm{N}$-terminal neutral tyrosine as electron donor. The positive charge on the C-terminal amino acid was introduced by photoinduced Norrish cleavage of ketone 2, subsequent phosphate elimination $3 \rightarrow 4$, and oxidation of the aromatic system $4 \rightarrow 5$. $^{38-41}$

In the middle between donor and acceptor an amino acid with natural or artificial side chain $\mathrm{X}$ was introduced, and these three amino acids were separated from each other by proline bridges that introduce a polyproline II (PPII) helical structure. ${ }^{44}$ Amino acids whose side chains $\mathrm{X}$ can be oxidized by the electron acceptor 5 are relay amino acids that act as stepping stones. For $\mathrm{X}=2,4,6$-trimethoxyphenyl the ET occurs as a two-step reaction, and the absolute rates of the individual steps could be determined in time-resolved laser experiments: 

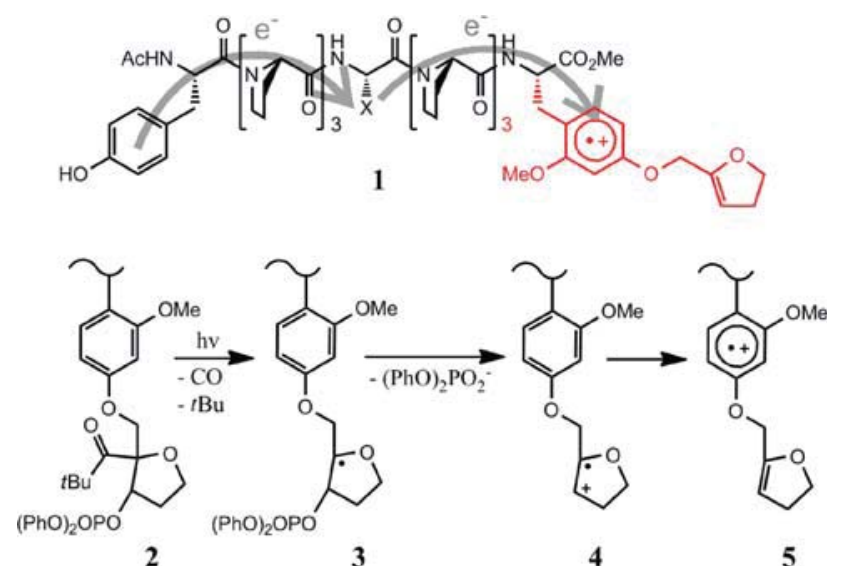

Figure 1. Model peptide for ET studies. The C-terminal non-natural aromatic amino acid residue contains a photoexcitable 2-(2,2dimethyl-propionyl)-3-(diphenoxyphosphoryloxy)tetrahydrofuran-2ylmethyl group in the 4-position that forms a tetrahydrofuran radical cation 4 upon UV exposure. If $\mathrm{X}$ can be oxidized by the electron acceptor at the C-terminal amino acid, ET occurs in a two step reaction as indicated in 1 . The electron acceptor is generated by photoinduced cleavage of ketone 2 , subsequent phosphate elimination $3 \rightarrow 4$, and oxidation of the aromatic group $4 \rightarrow 5$. The synthesis and reaction mechanism of this site-specific charge injection system is described in detail in refs 23-26. In the molecule shown, after photoexcitation an electron would transfer from the $\mathrm{N}$-terminal tyrosine (left) to the C-terminal electron acceptor group (right). Various amino acid side chains $\mathrm{X}$ can be introduced in the middle of the helix to determine their effect on the ET. In our simulations, the bridging group $\mathrm{X}$ was set to one of the five natural amino acid side chains phenylalanine, tyrosine, tryptophan, cysteine, and methionine.

the radical cation at the C-terminal amino acid of 1 was generated by laser irradiation of the ketone precursor 2 . The first ET step was oxidation of the 2,4,6-trimethoxyphenyl side chain ( $\mathrm{X}$ in 1), which generated its radical cation. The second step was a proton coupled ET from the tyrosine leading to a tyrosyl radical. All three reactive intermediates could be observed, and their concentration change followed by UV/vis spectroscopy. This made the rate determination of this consecutive reaction kinetics possible. ${ }^{41}$ Since ET can be photoinduced selectively and intermediates are observable by fast spectroscopic methods, this peptide assay offers excellent properties to study biochemical ET under defined conditions.

ET between the two terminal amino acid side chains of the peptide occurs roughly within a submicrosecond time frame, with strong dependence on the nature of the bridging amino acid $\mathrm{X}^{38}$ Transfer rates have been calculated for various derivatives of the peptide in Figure 1 with a bridging group $\mathrm{X}$ of 2,4,6-trimethoxyphenyl (Table 1). The experimental setup used ultrafast laser spectroscopy to simultaneously record the transient absorption spectra of the different cations (e.g., for the tyrosyl radical at $\lambda_{\max }=408 \mathrm{~nm}$ or for the trimethoxyphenylalanine radical at $\lambda_{\max }=550 \mathrm{~nm}$ ) from 30 to $500 \mathrm{~ns}$ after starting the reaction. ${ }^{41}$ Radical concentrations and intramolecular reaction rates were determined via numerical curve analysis, ${ }^{42,43}$ assuming monoexponential rates but correcting for the competing intermolecular ET. For peptides bearing other bridging groups $\mathrm{X}$, no explicit rates have been calculated, instead refs 39 and 40 (see Table 1 in each) give qualitative information on the suitability of $\mathrm{X}$ to facilitate ET. The experimental setup determined the transient UV absorption at $40 \mathrm{~ns}$ after the initial laser flash charge injection
Table 1. Experimentally Determined Electron Transfer Rates for Various Peptide Systems with a Bridging Group $X$ of 2,4,6-Trimethoxyphenyl ${ }^{a}$

\begin{tabular}{|c|c|c|c|}
\hline C-terminus & $\mathrm{N}$-terminus & $k_{\mathrm{D} \rightarrow \mathrm{X}}$ & $k_{\mathrm{X} \rightarrow \mathrm{A}}$ \\
\hline \multicolumn{4}{|c|}{ C-terminal acceptor, $\mathrm{N}$-terminal donor } \\
\hline $\mathrm{CO}_{2} \mathrm{Me}$ & NHAc & $(3.0 \pm 1.3) \cdot 10^{7} s^{-1}$ & $(3.3 \pm 0.5) \cdot 10^{6} s^{-1}$ \\
\hline $\mathrm{CO}_{2} \mathrm{Me}$ & $\mathrm{NH}_{3}^{+}$ & $(7.5 \pm 1.8) \cdot 10^{6} s^{-1}$ & $(2.5 \pm 0.3) \cdot 10^{6} s^{-1}$ \\
\hline \multicolumn{4}{|c|}{ C-terminal donor, $\mathrm{N}$-terminal acceptor } \\
\hline $\mathrm{CO}_{2} \mathrm{Me}$ & NHAc & n.d. & $(1.3 \pm 0.1) \cdot 10^{6} s^{-1}$ \\
\hline $\mathrm{CO}_{2} \mathrm{Me}$ & $\mathrm{NH}_{3}^{+}$ & n.d. & $(1.4 \pm 0.1) \cdot 10^{7} s^{-1}$ \\
\hline
\end{tabular}

and determined the amount of donor tyrosyl radical cation accumulated at this point through intramolecular ET, again correcting for competing intermolecular reactions. The latter studies showed that side chains that are comparably easy to oxidize, like tryptophan, methionine or di- and trimethoxy phenylalanine, speed up the overall ET rate between the peptide termini considerably, unlike bridging groups alanine, leucine or phenylalanine.

Open questions remain about the peptide structure in solution once a cation is formed (it is assumed to still be a stable PP-II helix) and about the exact mechanism of ET. Direct through-space ET via vacuum tunneling between donor and acceptor is strongly distance dependent and not relevant in the case here, where a condensed medium (both peptide and surrounding solvent) separates donor and acceptor. Conceivable transport mechanisms involve direct superexchangemediated tunneling between donor and acceptor, intermediate charge transfer to the bridging group $\mathrm{X}$ or via localized states on segments of the proline bridge. ${ }^{45,46}$ This will depend on the properties of the intermediate X-functionality as well as the energetics and conformations of the proline bridge. The ET mechanism is also influenced by the conformation dependent efficiency of through-bond electronic coupling involving the peptide backbone. ${ }^{47,48}$ Furthermore, the influence of solvent molecules on the stabilization and mobility of charges is unclear. These questions all involve atomistic details of the system dynamics and energetics, and molecular simulation methods are uniquely suited to address them.

In this study, we will present extensive molecular dynamics (MD) simulations of solvated model peptide systems and compute charge transfer parameters according to Marcus theory of ET. ${ }^{49-51}$ We will build upon our previous work on describing ET by computer simulation methods. We have previously argued ${ }^{52}$ that theoretically determining all three Marcus parameters involves multiscale modeling, since the electronic coupling $H_{\mathrm{DA}}$ is typically determined from quantum mechanical calculations, while the reaction free energy $\Delta G^{0}$ and reorganization energy $\lambda$ require thermodynamic averaging over a sufficiently large conformational ensemble, best done by molecular dynamics simulations. The background and methods used have been presented elsewhere in detail, ${ }^{53-56}$ and we will outline them only briefly in the following before turning to a discussion of the simulation results.

\section{MODELS AND METHODS}

Molecular Dynamics Simulations and Model Building. All MD simulations were conducted using version 4.5 of the Gromacs MD engine. ${ }^{57,58}$ For the NPT ensemble, temperature and pressure conditions were controlled by a Berendsen coupling algorithm. The Particle Mesh Ewald model with a 10 
Å direct space cutoff was used for long-range electrostatics, with the same cutoff for van der Waals interactions. A time step of 2 fs was used while constraining bonds involving hydrogen atoms using the Lincs algorithm. ${ }^{59}$ All data collection MD simulations were preceded by a standard equilibration protocol involving 100 steps of energy minimization, a 20 ps temperature equilibration to $300 \mathrm{~K}$ in the NVT ensemble and a final 400 ps NPT density equilibration. All visualization was conducted using VMD. ${ }^{60}$

Peptide models were built as Polyproline II helices in molden $^{61}$ (setting the backbone $\phi$ and $\psi$ angles to $-75^{\circ}$ and $+145^{\circ}$, respectively), parametrized according to the Amber99SB all-atom force field ${ }^{62}$ and embedded in a $10 \AA$ deep layer of TIP4P water molecules. ${ }^{63,64} \mathrm{~A}$ total of five different peptide systems were investigated, using phenylalanine, tyrosine, tryptophan, cysteine and methionine as central amino acid residue $\mathrm{X}$ in Figure 1. The $\mathrm{C}$ - and N-terminal donor and acceptor amino acid residues (AAR) were both modeled as tyrosine, to avoid uncertainties from introducing new parameters for a dialkoxy phenylalanine residue. Each model was built in four different charge states: neutral and with positively charged donor, acceptor and bridging residues. In the following, we define donor and acceptor with respect to a moving electron hole, a state $\mathrm{AXD}^{+}$for example symbolizes the initial condition of a positive charge introduced at the $\mathrm{C}$ terminus.

Atomic partial charges for radical cation species were determined by calculating RESP partial charges for neutral and charged hydrogen-capped side chain analogues. ${ }^{65-67}$ The differences in these two charge sets were then added to the atomic partial charges of the amino acid side chain model, to maintain consistency with the original force field charge parametrization protocol.

Electron Transfer Rates. The goal of the presented simulations is to study the mechanism of charge transfer, based on an atomistic model of the peptide system (Figure 1). Electron transfer rates are calculated according to Marcus' seminal theory, in which nonadiabatic charge transfer is envisioned as a two-state process with a charge moving between an initial donor and a final acceptor. The rate of this process is governed by three parameters: the thermodynamic driving force $\Delta G^{0}$, the coupling between donor and acceptor $H_{\mathrm{DA}}$ and the reorganization energy $\lambda$, equal to the energy needed to transform the system coordinates into the final state without charge transfer taking place. The transfer rate is then given by

$$
k_{\mathrm{ET}}=\frac{H_{\mathrm{DA}}{ }^{2}}{\hbar} \sqrt{\frac{\pi}{\lambda k_{\mathrm{B}} T}} \exp \left[-\frac{\left(\Delta G^{0}+\lambda\right)^{2}}{4 \lambda k_{\mathrm{B}} T}\right]
$$

Equation 1 has been extensively applied in charge transfer theory and remains the cornerstone of its theoretical description, even though multiple extensions and improvements have been developed over the years. ${ }^{68-70}$ Even in cases where the underlying assumptions of weak donor-acceptor coupling, classical harmonic nuclear motions, and the BornOppenheimer approximation become questionable and rates according to eq 1 therefore become unreliable, the three parameters still serve as elementary descriptors of a charge transfer system.

The semiclassical eq 1 relies on the validity of the FranckCondon (FC) approximation. The importance of non-Condon effects in dynamical charge transfer systems has been discussed recently. ${ }^{71-74}$ For an estimation on the importance of nonCondon effects in the studied system, we will follow the approach outlined in ref 71 where the validity of the FC approximation is judged from the relative magnitude of two decay times, that of the FC overlap factor $\tau_{\mathrm{FC}}$, and that of the electronic matrix element $\tau_{H_{\mathrm{DA}}}$. While $\tau_{\mathrm{FC}}$ can be directly calculated from classical simulations, ${ }^{75-78}$ we will use the expression for its semiclassical limit, $\tau_{\mathrm{FC}}=\bar{h}\left(2 \lambda k_{\mathrm{B}} T\right)^{1 / 2} \cdot{ }^{79,80}$ Using reorganization energies in the range of $1.8-2.3 \mathrm{eV}$ (Table 3 ) we obtain $\tau_{\mathrm{FC}} \approx 2$ fs. To calculate the decay time of the electronic matrix element, a short 1 ps MD simulation was conducted for the peptide carrying tyrosine as charged bridging group $\mathrm{X}$, using a randomly selected starting conformation. The couplings $H_{\mathrm{DA}}$ were computed as described below for $1000 \mathrm{MD}$ snapshots, spaced $1 \mathrm{fs}$ appart, as well as the corresponding autocorrelation function $C_{\mathrm{H}_{\mathrm{DA}}}{ }^{81} \tau_{\mathrm{H}_{\mathrm{DA}}}$ was found to be $29 \mathrm{fs}$ from a single exponential fit of $C_{H_{\mathrm{DA}}}$ up to a maximum time lag of 300 fs. Repeating the calculation for nine different starting conformations yielded similar or larger results for $\tau_{\mathrm{H}_{\mathrm{DA}}}$.

Since the electronic matrix element decay time $\tau_{H_{\mathrm{DA}}}$ is an order of magnitude larger than $\tau_{\mathrm{FC}}$, non-Condon effects should play a minor role in the studied system. This can be further quantified by computing the ratio of the static limit rate constant (Marcus rate) $k_{0}$ and its first nonzero correction term $k_{2}$ from perturbation theory. ${ }^{71,73,82}$ Using eq 40 in ref 73 , we obtain $k_{2} / k_{0}<0.1$, and eq 1 was used in the following to compute rates without further corrections.

A simpler, empirical electron transfer rate formula, assuming activationless ET, has been widely applied for proteins. ${ }^{4,83,84}$ In it, the rate is modeled by a simple exponential decay function of the form

$$
k_{\mathrm{ET}}=k_{0} \exp \left(-\beta r_{\mathrm{DA}}\right)
$$

where $k_{0}$ is a distance-independent prefactor that gives a rate of $10^{13} \mathrm{~s}^{-1}$ at van der Waals contact distance ${ }^{83}$ and $\beta$ is a distance decay factor that depends on the nature of the matrix in which the charge transfer sites are embedded. A value of $\beta=1.4 \AA^{-1}$ was originally suggested for proteins, while more accurate estimates also take the protein secondary structure into account. $^{36,85-87}$ A more recent study by Jones et al. that reexamined the decay parameter based on 28 protein structures and the empirical tunneling pathway model gives a range of 1.0-1.5 $\AA^{-1}$ for typical $\beta$ values in proteins, ${ }^{88}$ which has consistently been used in several studies. ${ }^{89-93}$

Determining Marcus Parameters. To compute $\Delta G^{0}$, the free energy difference between the two potential curve minima in Figure 2, we conceptually split it into two contributions, an internal one connected to the electronic structure of donor and acceptor and an external one dealing with interactions with the environment. The reaction free energy for charge transfer between the initial and the final state $\mathrm{D}^{+} \mathrm{A}$ and $\mathrm{DA}^{+}$(for ET involving the bridging group, either $\mathrm{D}$ or $\mathrm{A}$ would be replaced by $\mathrm{X}$ ) is then calculated as:

$$
\Delta G^{0}=\Delta \mathrm{IP}_{\mathrm{DA}}+\left\langle E_{\mathrm{DA}^{+}}^{\mathrm{FF}}\right\rangle_{\mathrm{DA}^{+}}-\left\langle E_{\mathrm{D}^{+} \mathrm{A}^{\mathrm{FF}}}\right\rangle^{+} \mathrm{A}
$$

where $\Delta \mathrm{IP}$ is the difference in vacuum ionization potential of the residues carrying the positive charge and $E^{\mathrm{FF}}$ is the total, classical force field energy of a state with angular brackets representing Boltzmann weighted ensemble averages using the potential energy function indicated in the subscript. The $E^{\mathrm{FF}}$ terms were corrected to remediate the effect of changing 


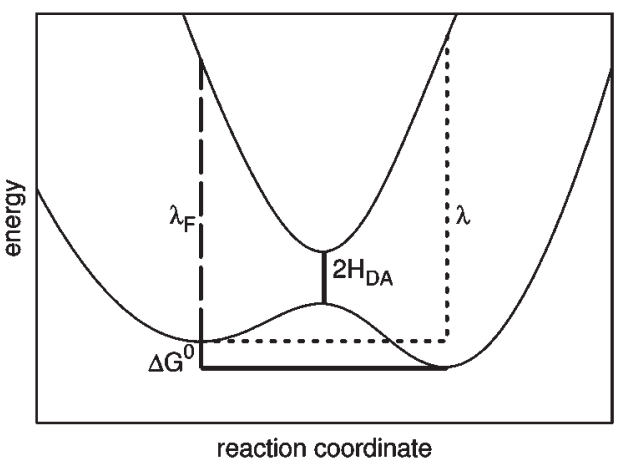

Figure 2. Scheme of the Marcus model. The transfer parameters $\Delta G^{0}$ (solid line), $\lambda$ (dotted line) and $H_{\mathrm{DA}}$ govern the ET rate. The vertical excitation energy for the forward transfer, $\lambda_{\mathrm{F}}$ (dashed line) is also shown. For $\Delta G^{0}=0$, the reorganization and excitation energies become equal, $\lambda_{\mathrm{F}}=\lambda$.

nonphysical internal force field terms that do not contribute to the external part of $\Delta G^{0}$.

As is common in ET theory, the reorganization energy $\lambda$ was likewise split into two components to facilitate computation, an internal $\lambda_{\mathrm{i}}$ and a $\lambda_{\mathrm{s}}$ for the effect of the surrounding

$$
\lambda_{\mathrm{s}}=s_{\mathrm{Pol}}\left(\left\langle E_{\mathrm{D}^{+} \mathrm{A}}^{\mathrm{FF}}\right\rangle_{\mathrm{DA}^{+}}-\left\langle E_{\left.\mathrm{D}^{+} \mathrm{A}^{\mathrm{FF}}\right\rangle_{\mathrm{D}^{+} \mathrm{A}}}\right)\right.
$$

where $E^{\mathrm{FF}}$ again stands for a classical total energy computed using the molecular dynamics force field and $s_{\mathrm{Pol}}$ is a scaling factor correcting for electronic polarization effects that are neglected in a point charge force field. Ignoring polarization and using unscaled computed reorganization energies yields values that are far to high. Therefore, a scaling factor $s_{\text {Pol }}$ of $1 / 2$ was used to correct $\lambda_{\mathrm{S}}$. The neccessity for this correction has been discussed before, ${ }^{94-97}$ as well as the approximation of uniform scaling factors of 0.5 to 0.7 for a complex protein/ solvent system (equivalent to assuming a uniform optic dielectric constant $\varepsilon_{\infty}$ of $\left.1.4-2\right) .^{98-102}$

In contrast to the external reorganization energy $\lambda_{\mathrm{s}}$, the internal $\lambda_{\mathrm{i}}$ mainly contains the effect of the changing electronic structure of donor and acceptor and is accessible via quantum mechanical calculations: ${ }^{54,103-109}$

$$
\lambda_{\mathrm{i}}=E_{\mathrm{D}}^{\mathrm{QM}}\left(\mathrm{D}^{+}\right)+E_{\mathrm{A}^{+}}^{\mathrm{QM}}(\mathrm{A})-E_{\mathrm{D}^{+}}^{\mathrm{QM}}\left(\mathrm{D}^{+}\right)-E_{\mathrm{A}}^{\mathrm{QM}}(\mathrm{A})
$$

Here, $E^{\mathrm{QM}}$ stands for the single point energy of the molecule in brackets, using the optimized geometry of the molecule in subscript. The molecules were generated by cutting the $\mathrm{C}_{\alpha}-\mathrm{C}_{\beta}$ bond of the AAR and saturating with a hydrogen atom. Geometry optimization and energy calculations were conducted using DFT ${ }^{110,111}$ with the B3LYP/6-311g $(2 \mathrm{~d}, 2 \mathrm{p})$ level of theory. ${ }^{112-115}$

There are multiple ways to calculate the coupling matrix elements $H_{\mathrm{DA}}{ }^{4,47,116,117}$ A fast and efficient way to obtain them is from the donor and acceptor HOMO wave functions of the neutral systems in the fragment orbital approach (FMO $)^{55,118}$

$$
H_{\mathrm{DA}}=\int_{\tau} \psi_{\mathrm{D}}^{\mathrm{HOMO}^{\mathrm{H}}} \psi_{\mathrm{A}}^{\mathrm{HOMO}} d \tau
$$

In this study, we used the approximate density functional theory method DFTB to compute electronic couplings. ${ }^{19,120}$ DFTB in combination with the FMO approach in eq 6 has been used in our group to compute electronic couplings $H_{\mathrm{DA}}$ in proteins and DNA before. ${ }^{53,54,56,121,122}$ The method was shown to provide $H_{\mathrm{DA}}$ values in good agreement to high-level ab initio results in extensive benchmarking calculations. ${ }^{55}$ As our calculations consider fluctuations in the coupling matrix element, $H_{\mathrm{DA}}$ in eq 1 needs to be replaced by an averaged coupling. For the rates calculated below, $\left\langle\left. H_{\mathrm{DA}}\right|^{2}\right\rangle$ was used.

An alternative, empirical approach to computing electronic couplings from geometrical considerations is the pathway model. ${ }^{123,124}$ In it, the donor-acceptor coupling $H_{\mathrm{DA}}$ is expressed as the product of a contact coupling $H_{\mathrm{DA}}^{\text {contact }}$ between donor and acceptor in close proximity and a distance decay factor $\varepsilon$. This factor $\varepsilon$ is the product of multiple atom-to-atom decay factors that take into account the location of bonds and hydrogen bonds in the system. For each possible pathway between donor and acceptor, an $\varepsilon$ value can be computed, and the highest value corresponds to the optimal electron transfer path between donor and acceptor

$$
\begin{aligned}
& H_{\mathrm{DA}}=\varepsilon H_{\mathrm{DA}}^{\text {contact }} \\
& \varepsilon=\prod_{i} \varepsilon_{i} \\
& \varepsilon_{\text {bond }}=0.6 \\
& \varepsilon_{\mathrm{H}-\text { bond }}=0.36 \exp [-1.7(R-2.8)] \\
& \varepsilon_{\text {vacuum }}=0.6 \exp [-1.7(R-1.4)]
\end{aligned}
$$

The product runs over all atom-to-atom steps of a particular pathway and the $\varepsilon_{i}$ differ depending on the atom connectivity (parameters from ref 124 were used here). $R$ is the atom-toatom distance.

All parameters necessary to calculate Marcus rates according to eq 1 are therefore easily accessible from MD simulations or straightforward QM calculations. Thus, computationally predicted ET rates can be compared to experimental data to judge if simulation results give an accurate microscopic picture of the studied CT phenomena. It should be noted that even moderate errors introduced when calculating the Marcus parameters can lead to order of magnitude disagreement of theoretical and experimental rates, nevertheless the parameters themselves can shed light on interesting system properties. So, qualitative predictions, e.g., on the possible CT mechanisms like single step or multistep hopping, can often be made.

Our approach to calculate Marcus parameters relies on classical MD simulations to obtain components of the reorganization energy and reaction free energy. This is necessary, since $\lambda_{\mathrm{s}}$ and the external part of $\Delta G^{0}$ are dependent on a correct Boltzmann weighted ensemble averaging of all relevant conformations, which requires fast empirical methods due to the enormous number of degrees of freedom in a solvated system. The computation of reorganization energies for complex solvated systems has been a subject of considerable interest over the last years. As an alternative to our approach, ab initio molecular dynamics simulations have been popular due to their ability to treat electronic and nuclear degrees of freedom simultaneously. ${ }^{125-130}$ For the treatment of larger systems, a $\mathrm{QM} / \mathrm{MM}$ formalism is normally introduced to avoid large scale QM calculations by obtaining conformational snapshots from classical MD simulations and reducing parts of the system like the solvent to a classical point charge or continuum electrostatics description. ${ }^{95,131-134}$ Then, electronic polar- 

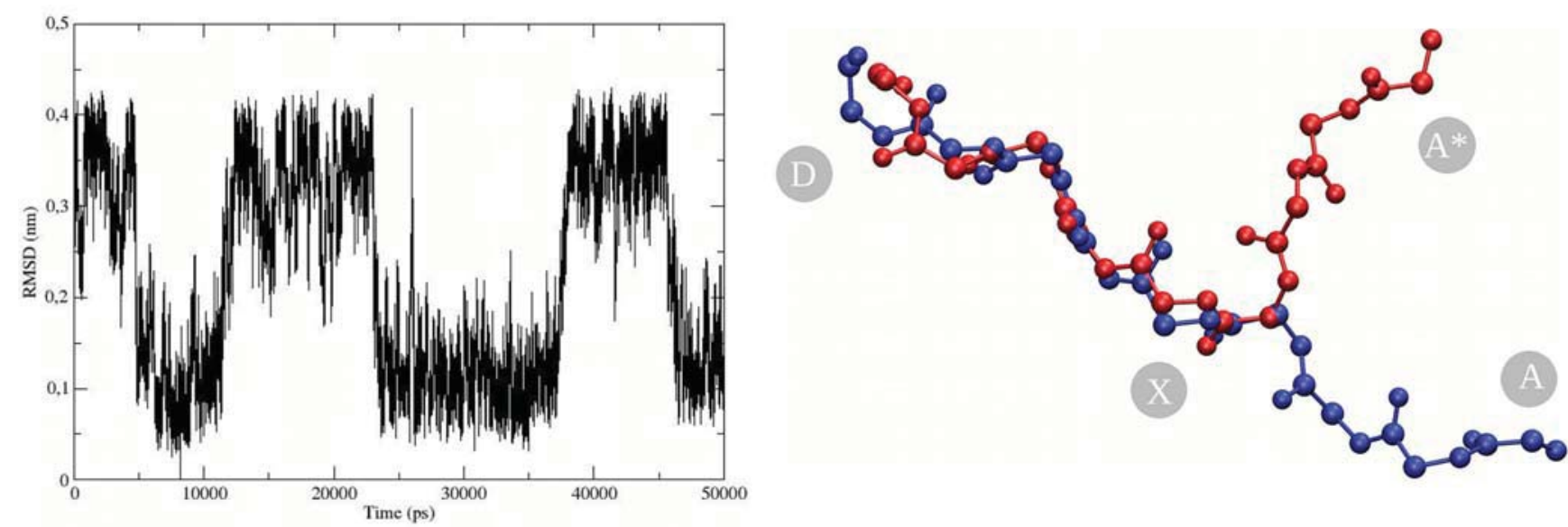

Figure 3. Typical peptide $\mathrm{C}_{\alpha}$ RMSD values over $50 \mathrm{~ns}$. Data shown is for the neutral DXA case with $\mathrm{X}=\mathrm{Trp}$. Two alternating main conformations can be distinguished. RMSD values are computed with respect to the ideal starting structure. The two conformations correspond to an unperturbed PPII helix (blue) and a slightly kinked helix (red). The kink occurs between residues 4 and 5. Gray circles indicate the approximate positions of the center of mass for the charge carrying amino acid side chains donor D, central $\mathrm{X}$ and acceptor A. Note that while the position of the acceptor changes significantly when the helix is kinked (to that of $\mathrm{A}^{*}$ ), the distance between $\mathrm{X}$ and A remains nearly constant.

ization effects can be taken into account via constant scaling schemes, as discussed above. . $^{96-102}$

Our approach to separate the reorganization energy $\lambda$ into internal contributions $\lambda_{i}$, which can be determined by single point QM calculations, and external contributions $\lambda_{s}$ from classical MD simulations provides computational efficiency by maintaining a QM description only where necessary. This allows an extensive sampling of conformational space in multinanosecond $\mathrm{MD}$ simulations, which is a requirement to accurately describe the structure and dynamics of flexible biomolecules. A precondition is the existence of a reasonable boundary between localized electron transfer sites, identified here as the $\pi$-electron systems of amino acid side chains, and their classically described environment. While ab initio molecular dynamics simulations avoid such an artificial partitioning of the system and may be desirable for smaller molecules, we believe that a $\mathrm{QM} / \mathrm{MM}$ treatment as outlined above is currently the optimal way of describing ET in large biomolecules.

\section{RESULTS}

Peptide Structure and Dynamics. To obtain a sufficiently large conformational ensemble for parameter evaluation, an unrestrained $50 \mathrm{~ns}$ length $\mathrm{MD}$ simulation was conducted for each of the five studied peptides in all four possible charge states (DXA, $\mathrm{D}^{+} \mathrm{XA}, \mathrm{DX}^{+} \mathrm{A}, \mathrm{DXA}^{+}$). All simulations started from a preequilibrated polyproline II helix structure, and the overall helical shape of each studied peptide was maintained during the simulations. However, $\mathrm{C}_{\alpha}$ rmsd plots suggested the existence of at least two main conformational states (Figure 3). A clustering analysis (using the single linkage method of the Gromacs $g$ _cluster tool) revealed that the majority of structures in the conformational ensemble fell into two main clusters: The average structure of the first important conformation is an extended polyproline II helix very similar to the idealized starting conformation (average rmsd less than $0.25 \mathrm{~nm}$ ) and the second one represents a slightly kinked helix, bent at the center by about $60^{\circ}$. Interestingly, the occurrence of the kinked conformation is strongly dependent on the nature of the bridging AAR $X$, but not significantly on the charge state of the system (Table 2). For Phe and Tyr as bridging residues, the
Table 2. Probability of Finding the Helix in Either the Linear or Slightly Bent Conformation Is Strongly Influenced by the Nature of the Bridging AAR $\mathrm{X}$

\begin{tabular}{ccc} 
X & \% PPII & \% kinked \\
Phe & 53 & 25 \\
Tyr & 61 & 23 \\
Trp & 48 & 32 \\
Met & 78 & 3 \\
Cys & 67 & 14 \\
\hline
\end{tabular}

straight PPII helix prevails, for Trp both conformations occur frequently, and for the sulfur-containing AAR, kinked helices are observed only rarely. The percentages in Table 2 are subject to large standard deviations of $20 \%$ or more though, meaning that for almost all charge states and bridging residues, at least one simulation is found where either the kinked or the linear conformation occurs predominantly. From a visual analysis of rmsd values, approximately 54 conformational transitions were observed in total, corresponding to an average lifetime of 18.5 ns for one conformational substate. Since only about 1 to 3 transitions were observed in every individual simulation, the numbers presented in Table 2 should be discussed only qualitatively and much more sampling would be needed for quantitative statistics. It should be noted that this conformational interconversion of the peptide structures occurs on a much more rapid time scale than the ET reactions, so analyzing reaction rates under the assumption of a monoexponential rate constant, as done in ref 41 appears to be valid. Still, we can conclude that the peptide maintains a rigid helical structure regardless of the presence and location of a positive charge in the system. In several cases depending on the exact sequence, a second conformation is frequently observed and may influence charge transfer properties.

As charge transfer is a strongly distance-dependent process, the second important piece of information extracted from the simulation results are the distance distributions between donor, acceptor and bridging residues. Interestingly, these distances do not change very much between both conformations, since the bending point of the helix is close to the position of the central AAR X. We obtained average distances from all $20 \mathrm{MD}$ 
simulations of $R_{\mathrm{D}-\mathrm{X}}=12.8 \pm 0.5 \AA$ and $R_{\mathrm{X}-\mathrm{A}}=13.0 \pm 0.6 \AA$. Conversely, the donor-acceptor distance itself is influenced by the conformational change: an average of $R_{\mathrm{D}-\mathrm{AP}}^{\mathrm{PPII}}=21.5 \pm 0.6 \AA$ is found for the simulations with mostly linear helices, whereas a $R_{\mathrm{D}-\mathrm{A}}^{\text {kined }}=19.8 \pm 0.5 \AA$ distance is found when averaging over the MD simulations in which the helix is mainly kinked. Note that the standard deviations do not represent error estimates but rather the width of the natural conformational ensemble caused by thermal fluctuation.

Charge Transfer Parameters. We now turn to computing the Marcus parameters of charge transfer from the conformational ensembles presented so far. Table 3 summarizes the

\section{Table 3. Calculated Marcus Parameters ${ }^{a}$}

\begin{tabular}{cccccrrr}
$\mathrm{X}$ & transfer & $\lambda_{\mathrm{i}}$ & $\lambda_{\mathrm{s}}$ & $\lambda$ & $\Delta E^{\text {Env }}$ & \multicolumn{1}{c}{$\Delta \mathrm{IP}$} & \multicolumn{1}{c}{$\Delta G^{0}$} \\
Phe & $\mathrm{D} \rightarrow \mathrm{X}$ & 0.38 & 1.69 & 2.07 & 0.50 & 0.64 & 1.14 \\
& $\mathrm{X} \rightarrow \mathrm{A}$ & 0.38 & 1.55 & 1.93 & -0.52 & -0.64 & -1.16 \\
& $\mathrm{D} \rightarrow \mathrm{A}$ & 0.42 & 1.78 & 2.20 & -0.02 & 0.00 & -0.02 \\
Tyr & $\mathrm{D} \rightarrow \mathrm{X}$ & 0.42 & 1.62 & 2.04 & -0.03 & 0.00 & -0.03 \\
& $\mathrm{X} \rightarrow \mathrm{A}$ & 0.42 & 1.65 & 2.07 & -0.04 & 0.00 & -0.04 \\
& $\mathrm{D} \rightarrow \mathrm{A}$ & 0.42 & 1.76 & 2.18 & -0.07 & 0.00 & -0.07 \\
Trp & $\mathrm{D} \rightarrow \mathrm{X}$ & 0.38 & 1.58 & 1.97 & 0.28 & -0.23 & 0.05 \\
& $\mathrm{X} \rightarrow \mathrm{A}$ & 0.38 & 1.47 & 1.84 & -0.32 & 0.23 & -0.09 \\
& $\mathrm{D} \rightarrow \mathrm{A}$ & 0.42 & 1.76 & 2.18 & -0.05 & 0.00 & -0.05 \\
Met & $\mathrm{D} \rightarrow \mathrm{X}$ & 0.28 & 1.81 & 2.10 & -0.04 & 0.46 & 0.42 \\
& $\mathrm{X} \rightarrow \mathrm{A}$ & 0.28 & 1.67 & 1.96 & 0.08 & -0.46 & -0.38 \\
& $\mathrm{D} \rightarrow \mathrm{A}$ & 0.42 & 1.77 & 2.19 & 0.04 & 0.00 & 0.04 \\
Cys & $\mathrm{D} \rightarrow \mathrm{X}$ & 0.26 & 2.03 & 2.29 & -0.32 & 1.05 & 0.73 \\
& $\mathrm{X} \rightarrow \mathrm{A}$ & 0.26 & 1.83 & 2.09 & 0.40 & -1.05 & -0.65 \\
& $\mathrm{D} \rightarrow \mathrm{A}$ & 0.42 & 1.82 & 2.24 & 0.08 & 0.00 & 0.08
\end{tabular}

${ }^{a}$ All energies given in $\mathrm{eV}$. A scaling factor of $1 / 2$ was used for $\lambda_{s}$.

results obtained. For the reorganization energy $\lambda$, the breakdown between the internal part $\lambda_{\mathrm{i}}$ and the external part $\lambda_{\mathrm{s}}$ is given. Likewise, for $\Delta G^{0}$ both the internal components $\Delta \mathrm{IP}$ and the external $\Delta E^{\mathrm{Env}}$ is given. Both quantities contain an internal and an external component, computed quantum mechanically and classically, respectively.

While adiabatic IP values can be determined experimentally, ${ }^{135}$ the vertical IP is more difficult to determine and is typically computed by ab initio methods. ${ }^{136}$ Here, we use data from Dehareng et al., ${ }^{137}$ in which IP values were obtained for multiple amino acid backbone and side chain conformations using the outer-valence Green's function (OVGF) method using DFT. We have used the average of the IP value range from multiple conformations in Table 4 of ref 137 to calculate the $\Delta \mathrm{IP}$ values in Table $3 . \Delta \mathrm{IP}$ gives the vertical ionization energy in vacuum without further relaxation or consecutive reactions as all environmental effects are contained in $\Delta E^{\mathrm{Env}}$. A scaling factor $s_{\text {Pol }}$ for $\lambda_{\mathrm{s}}$ was applied as described in eq 4 to account for electronic polarization effects. The $\lambda$ values reported are in good agreement to experimental values for comparable peptides. ${ }^{34}$

The calculated charge transfer parameters in Table 3 contain several noteworthy points. The internal reorganization energy makes up a small but noticeable portion of on average $18 \%$ of $\lambda$, but reorganization is dominated by the external, mostly solvent, $\lambda_{\mathrm{s}}$, as would be expected in a system where the charge carrying AAR are exposed to polar solvent. The total reorganization energy of on average $2.09 \mathrm{eV}$ is rather large for a biochemical system, again indicating the strong stabilization experienced by a charge on a fully solvated side chain. Before application of the scaling factor, $\lambda_{\mathrm{s}}$ values of ca. $3.5 \mathrm{eV}$ are obtained directly from the MD simulations. In comparison, significantly smaller unscaled values of ca. $2 \mathrm{eV}$ were found in our recent study of ET between AAR within a protein, ${ }^{54}$ but applying the same technique to ET between DNA nucleobases yielded $\lambda_{\mathrm{s}}$ values approaching $3 \mathrm{eV}$ for large transfer distances. ${ }^{138}$ Direct solvent exposure of charged side chains in peptides results in markedly different ET properties than would be typical for reactions occurring in the interior of a protein.

The $\lambda$ values are mostly unaffected by the nature of the bridging AAR and by the particular transfer selected. Even the direct $\mathrm{D} \rightarrow \mathrm{A}$ transfer over a much larger distance only shows a small increase of $0.16 \mathrm{eV}$ in $\lambda$ compared to $\mathrm{D} \rightarrow \mathrm{X}$ and $\mathrm{X} \rightarrow \mathrm{A}$ transfers. Since the transfers D $\rightarrow$ A always occur between Tyr residues and should not be influenced by the nature of $\mathrm{X}$, their standard deviation of $0.03 \mathrm{eV}$ gives an indication of the statistical uncertainty of the presented results.

The $\Delta \mathrm{IP}$ values show the part of the thermodynamic driving force due to the chemical nature of the bridging AAR. Note that the IP values used do not include the effect of protonation state changes after oxidation/reduction, in the same manner as our MD simulations, which also do not describe protonation reactions either. In good agreement to chemical intuition, only Trp has a lower redox potential than Tyr, while Phe and the sulfur-containing amino acids Met and Cys yield high energetic costs for donating electrons to Tyr. When the effect of the environment $\Delta E^{\mathrm{Env}}$ is taken into account, we obtain the overall energy necessary to localize a positive charge on the central AAR X. Three different cases can be distinguished: For Met and Tyr as X, the effect of the surrounding system is small; that is, these AAR are stabilized by the environment about as well as Tyr at the terminal positions of the peptide. For Tyr, this leads to the expected free energy difference of about zero for the whole transfer process, while for Met the cost of approximately $0.5 \mathrm{eV}$ caused by the different IP remains unchanged. The second case concerns Cys, which experiences considerably stronger environmental stabilization than the terminal Tyr residues. However, the large $\Delta \mathrm{IP}$ still results in a high energetic penalty that impedes charge localization on a central Cys residue. Finally, both for Trp and Phe a relative destabilization compared to the terminal AAR is obtained. This effectively counteracts the lower IP of Trp, leaving the charge transfer system Tyr-Trp-Tyr with a very small driving force, but for Phe both $\Delta E^{\mathrm{Env}}$ and $\Delta \mathrm{IP}$ combine to yield a high energetic barrier of over $1 \mathrm{eV}$.

Altogether, when $\Delta G^{0}$ values are obtained from combining $\Delta E^{\mathrm{Env}}$ and $\Delta \mathrm{IP}$, both effects strongly dependent on the exact nature of the bridging AAR. We suggest that bridging Tyr and Trp, and maybe to a lesser extent Met, could act as charge carrying residues, but for Cys and Phe this is unlikely due to the high energies involved.

When calculating the electronic couplings $H_{\mathrm{DA}}$ directly according to eq 6 for a series of MD snapshots, we obtained very small couplings in all cases. Absolute values are hard to ascertain due to well-known numerical instabilities. ${ }^{124}$ Nevertheless, we can give a conservative upper boundary of $1 \mathrm{meV}$ for $H_{\mathrm{DA}}$ in all cases when the intermediate medium is neglected. This approach has previously been used to compute electronic couplings between donor and acceptor molecules in closer contact, ${ }^{54-56}$ but it appears not suitable for the peptide systems studied here with larger $\mathrm{D}-\mathrm{A}$ distances.

Therefore, $H_{\mathrm{DA}}$ values were calculated with an alternative scheme according to the empirical eq 7 for the $\mathrm{D} \rightarrow \mathrm{X}$ and $\mathrm{X} \rightarrow$ 
Table 4. Average Electronic Couplings and Standard Deviations Calculated Using the Empirical Pathway Model ${ }^{a}$

\begin{tabular}{|c|c|c|c|c|c|c|c|}
\hline $\mathrm{X}$ & transfer & $\left|H_{\mathrm{DA}}^{\text {contact }}\right|$ & $\varepsilon^{\text {nowater }}$ & $\varepsilon^{\text {water }}$ & $\%$ water & $\left\langle\left|H_{\mathrm{DA}}\right|\right\rangle$ & $\left(\left\langle\left|\mathrm{H}_{\mathrm{DA}}\right|^{2}\right\rangle\right)^{1 / 2}$ \\
\hline \multirow[t]{2}{*}{ Tyr } & $\mathrm{D} \rightarrow \mathrm{X}$ & 0.24 & $(0.65 \pm 0.95) \cdot 10^{-3}$ & $(1.01 \pm 4.06) \cdot 10^{-3}$ & 16 & $2.40 \times 10^{-4}$ & $10.0 \times 10^{-4}$ \\
\hline & $\mathrm{X} \rightarrow \mathrm{A}$ & 0.24 & $(0.47 \pm 0.46) \cdot 10^{-3}$ & $(0.68 \pm 1.23) \cdot 10^{-3}$ & 20 & $1.63 \times 10^{-4}$ & $3.37 \times 10^{-4}$ \\
\hline \multirow[t]{2}{*}{$\operatorname{Trp}$} & $\mathrm{D} \rightarrow \mathrm{X}$ & 0.15 & $(1.46 \pm 4.92) \cdot 10^{-3}$ & $(1.91 \pm 5.20) \cdot 10^{-3}$ & 27 & $2.87 \times 10^{-4}$ & $8.31 \times 10^{-4}$ \\
\hline & $\mathrm{X} \rightarrow \mathrm{A}$ & 0.15 & $(0.51 \pm 0.59) \cdot 10^{-3}$ & $(1.04 \pm 2.34) \cdot 10^{-3}$ & 29 & $1.56 \times 10^{-4}$ & $3.84 \times 10^{-4}$ \\
\hline
\end{tabular}

${ }^{a}$ All couplings are given in eV. \% water indicates in which percentage of snapshots at least one water molecule was involved in the pathway.

A transfers in peptides containing Trp and Tyr as AAR X. The contact electronic couplings $H_{\mathrm{DA}}^{\text {contact }}$ were computed by placing the two molecules (as hydrogen saturated side chain analogs) in van der Waals contact distance with parallel $\pi$-electron systems and energy minimizing the complex before computing the coupling according to eq 6 using DFTB. Four rotational orientations of the two molecules were tested and the highest computed $H_{\mathrm{DA}}^{\text {contact }}$ was used in each case. All $H_{\mathrm{DA}}$ values were taken as positive. Optimal electron transfer pathways were determined for $500 \mathrm{MD}$ snapshots in each case, both with and without allowing water molecules to participate in a pathway. Table 4 summarizes the results.

For all four transfers studied, comparable couplings are obtained, with higher values for the $\mathrm{D} \rightarrow \mathrm{X}$ than for the $\mathrm{X} \rightarrow \mathrm{A}$ step for both central AAR. Water molecules are involved in the optimal pathway in $15-30 \%$ of cases. If water molecules are excluded from the pathways, significantly smaller $\varepsilon$-values are obtained. This indicates that the few pathways including water molecules tend to be the most efficient ones. In ca. 50\% of simulation snapshots, no better pathway than along the covalent linkage of the backbone is found.

The high standard deviations found for $\varepsilon$ indicate that ET is in the fluctuation-dominated regime as discussed by Skourtis et al. ${ }^{5,7}$ We obtain coherence parameter $C$ as defined in ref 7 of ca. 0.1. Therefore, rare fluctuations of the electronic coupling, driven by conformational changes and solvent dynamics, have an outsize influence on the charge transfer rate and due to the quadratic dependence of Marcus rates on $\left\langle H_{\mathrm{DA}}\right\rangle$, the more important parameter to consider is arguably $\left(\left\langle\mathrm{H}_{\mathrm{DA}}{ }^{2}\right\rangle\right)^{1 / 2}$. The final resulting average couplings are $0.21 \mathrm{meV}$ for $\left\langle\left|H_{\mathrm{DA}}\right|\right\rangle$ and $0.64 \mathrm{meV}$ for $\left(\left\langle\mathrm{H}_{\mathrm{DA}}{ }^{2}\right\rangle\right)^{1 / 2}$, confirming our previously estimated upper boundary of $1 \mathrm{meV}$ for the electronic coupling. In contrast, when couplings between the terminal donor and acceptor residues were calculated using the same pathway model, significantly smaller $\left\langle\left|H_{\mathrm{DA}}\right|\right\rangle$ values below $10^{-5} \mathrm{eV}$ were found, indicating that direct transfer between the peptide termini is not relevant here.

\section{DISCUSSION}

Charge transfer is known from experiment to proceed along the peptide helix in the time frame of approximately $50 \mathrm{~ns}$ if the bridging AAR is Trp, methoxy-substituted phenylalanine, Cys or Met. ${ }^{39}$ Experimental transfer rates in the range of $k_{\mathrm{r}} \approx 10^{6}-$ $10^{8} \mathrm{~s}^{-1}$ are available for different peptide substitutions. ${ }^{41}$ Since our model introduced identical donors and acceptors, the experimental value best to compare to would also be one in which transfer between almost isoenergetic sites was measured. For such a case, a reaction rate of $k_{\mathrm{R}}=(3.3 \pm 0.5) \cdot 10^{6} \mathrm{~s}^{-1}$ is given ( $k_{1}$ for molecule 10a in Scheme 4 of ref 41 ; see also Table 1).

Corresponding Marcus rates for individual $\mathrm{D} \rightarrow \mathrm{X}$ or $\mathrm{X} \rightarrow \mathrm{A}$ transfers with the parameters from Table 3 are orders of magnitude smaller than that. The largest rates are found to be below $10^{2} \mathrm{~s}^{-1}$ for the case of Tyr as $\mathrm{X}$ and even smaller for all other bridging AAR. Clearly there are tremendous uncertainties in all three Marcus parameters as computed here, which very strongly influence the resulting ET rates due to the exponential and quadratic dependence of $k_{\mathrm{r}}$ on $\lambda, \Delta G^{0}$ and $H_{\mathrm{DA}}$ in eq 1 . It is difficult to give accurate error estimates for any of the three Marcus parameters. For the case of $H_{\mathrm{DA}}$, it was shown that close range couplings computed by semiempirical DFTB typically differ no more than a factor of 2 from higher level QM calculations. ${ }^{55}$ We estimate that the simple empirical scheme to compute the pathway decay factor could introduce significant further errors for $H_{\mathrm{DA}}$, so that our calculated couplings may be off by up to an order of magnitude. The case for the external parts of $\lambda$ and $\Delta G^{0}$ computed by molecular dynamics makes error estimation even more difficult, since it has been shown before that the numerical inaccuracies of such results are much smaller than their systematic errors. ${ }^{139}$ Therefore, computing a standard error of the mean can significantly underestimate the true inaccuracy. With the additional problem of accounting for electronic polarization effects by a simple scaling scheme, we can estimate that both the computed $\lambda$ and $\Delta G^{0}$ may be inaccurate by several $\mathrm{kJ} / \mathrm{mol}$, or up to approximately $30 \%$. To obtain rates in the right order of magnitude for, e.g., Tyr, $\lambda_{s}$ would need to be reduced by $30 \%$ and the electronic coupling increased by a factor of 10 at the same time, yielding a $k_{\mathrm{D} \rightarrow \mathrm{X}}=2.6 \times 10^{6} \mathrm{~s}^{-1}$ and $k_{\mathrm{X} \rightarrow \mathrm{A}}=2.2 \times$ $10^{6} \mathrm{~s}^{-1}$, comparable to experiment but just on the border of a realistic parameter range in our simulations.

Therefore, we believe neither substantial inaccuracies in the calculated parameters, based on the semiempirical quantum method DFTB or the use of classical force fields, can alone account for the discrepancy, nor the small differences between the experimental and simulated systems (e.g., the use of chemically modified AAR in the experiment and measurements in a water:acetonitrile mixture as solvent).

However, several possible reasons for the disagreement between simulation and experiment can be given: First, experiments do not distinguish between electron transfer and coupled electron/proton transfer. Since, e.g., Tyr and Cys radical cations are strong acids, they can undergo a rapid deprotonation after oxidation. This would significantly alter the associated reaction free energies for the overall ET reaction. In our simulation, all protonation states remain unchanged, and the $\triangle I P$ values used for the AAR also do not take deprotonation into account. Furthermore, for the case of Met as AAR X, a high electron transfer efficiency is observed experimentally, but not predicted here. The fact that ET proceeds across a Met-bridge despite the high redox potential of a thioether, has been explained by the stabilizing neighboring group effect of adjacent amide functions. ${ }^{40} \mathrm{~A}$ molecular mechanics force field would not accurately model this effect. Finally, our simulation assumes full localization of the charge on either D, A or X at all times. Allowing partial charge delocalization between these sites or transient partial local- 
ization on other parts of the system could lower the barriers for ET and lead to higher predicted rates.

The rates found in the peptide system can also be compared to the empirical rate-distance relationship for electron transfer in proteins discussed by Moser, Hopfield et al. ${ }^{83,84}$ From their data (Table 1 in ref 83 ) a charge transfer rate of $k_{\mathrm{R}} \approx 10^{7}-$ $10^{9} \mathrm{~s}^{-1}$ would be expected for a protein system containing charge transfer sites at comparable distances. Electron transfer along the peptide helix apparently proceeds slower than it would embedded in a protein matrix. Possible reasons for this are the better stabilization of fully solvent exposed radical cations, higher conformational flexibility and weaker coupling between AAR with only a single helix connecting them. Since these factors would have little effect on the reaction rate prefactor $k_{0}$ in eq 2 , we conclude that the distance decay factor $\beta$, which was found to be about $1.4 \AA$ or smaller in proteins, appears to be larger in peptides, leading to overall slower transfer.

Even though eq 1 yields rates far too small to explain the observed charge transfer, the underlying parameters $\lambda, \Delta G^{0}$ and $H_{\mathrm{DA}}$ give a detailed picture of similarities and differences between systems differing in the bridging AAR X. Comparably large reorganization energies due to pronounced solvent stabilization of the charged side chains make charge transfer difficult in all cases, but differences in the chemical nature and solvation of side chains determine if charge transfer faces prohibitively high barriers. The extremely small through-space (fragment orbital based) direct electronic coupling $\left|H_{\mathrm{DA}}\right|$ indicates that either the peptide helix or intervening water molecules play an important role in mediating the donor/ acceptor interaction and taking the intervening medium into account using the pathway model results in a more realistic, but still small, coupling $H_{\mathrm{DA}}$. In comparison, assuming throughspace coupling between amino acid side chains at slightly smaller separation distance in the protein E. coli DNA photolyase or between neighboring nucleobases in DNA radical cations yielded ET rates in good agreement to experiment in recent studies. ${ }^{52,54,122,140,141}$

With direct ET between donor and acceptor ruled out, future studies of the system can focus on the role of the medium between two charge carrying side chains in more detail than the empirical pathway model allows. The peptide backbone may mediate charge movement along the PPII helix or even allow transient localization, and explicitly including these effects could lead to higher predicted transfer rates. Furthermore, it has been shown that bridging water molecules can serve to enhance electron transfer pathways in proteins, ${ }^{142,143}$ and a similar effect seems to be important in solvated peptides. This effect is already present in our results, seen in the importance of considering water molecules in the couplings in Table 4. To better capture such effects, it may be necessary to treat the interactions between moving charge and polarized environment in a more straightforward way, as was shown recently in coupled electron-nuclear dynamics QM/MM simulations. ${ }^{54,141}$

\section{AUTHOR INFORMATION}

\section{Corresponding Author}

*E-mail: thomas.steinbrecher@kit.edu.

\section{Notes}

The authors declare no competing financial interest.

\section{ACKNOWLEDGMENTS}

T.S. is indebted to the Baden-Württemberg Stiftung for the financial support of this research project by the Eliteprogramme for Postdocs.

\section{REFERENCES}

(1) Marcus, R. A. Adv. Chem. Phys. 1999, 106, 1-6.

(2) Hervas, M.; Navarro, J. A.; De la Rosa, M. A. Acc. Chem. Res. 2003, 36, 798-805.

(3) Bixon, M.; Jortner, J. Adv. Chem. Phys. 1999, 106, 35-202.

(4) Gray, H. B.; Winkler, J. R. Annu. Rev. Biochem. 1996, 65, 537561.

(5) Skourtis, S. S.; Waldeck, D. H.; Beratan, D. N. Annu. Rev. Phys. Chem. 2010, 61, 461-485.

(6) Beratan, D. N.; Skourtis, S. S.; Balabin, I. A.; Balaeff, A.; Keinan, S.; Venkatramani, R; Xiao, D. Acc. Chem. Res. 2009, 42, 1669-1678.

(7) Balabin, I. A.; Beratan, D. N.; Skourtis, S. S. Phys. Rev. Lett. 2008,

$101,158102$.

(8) Grozema, F. C.; Siebbeles, L. D. A.; Berlin, Y. A.; Ratner, M. A. ChemPhysChem 2002, 3, 536-539.

(9) Grozema, F. C.; Berlin, Y. A.; Siebbeles, L. D. A. J. Am. Chem. Soc. 2000, 122, 10903-10909.

(10) Vekhter, B. G.; Ratner, M. A. J. Chem. Phys. 1994, 101, 97109715.

(11) Vekhter, B. G.; Ratner, M. A. Phys. Rev. B 1995, 51, 3469-3475.

(12) Berlin, Y. A.; Siebbeles, L. D. A. Chem. Phys. Lett. 1998, 291, $85-93$.

(13) Siebbeles, L. D. A.; Berlin, Y. A. Chem. Phys. 1998, 238, 97-107.

(14) Olson, M.; Mao, Y.; Windus, T.; Kemp, M.; Ratner, M. A.; Leon, N.; Mujica, V. J. Phys. Chem. B 1998, 102, 941-947.

(15) Grozema, F. C.; vanDuijnen, P. T.; Berlin, Y. A.; Ratner, M. A.; Siebbeles, L. D. A. J. Phys. Chem. B 2002, 106, 7791-7795.

(16) Herz, T.; Gedeck, P.; Clark, T. J. Am. Chem. Soc. 1999, 121, 1379-1380.

(17) Warshel, A.; Parson, W. W. Q. Rev. Biophys. 2001, 34, 563-679.

(18) Warshel, A. Acc. Chem. Res. 2002, 35, 385-395.

(19) Small, D. W.; Matyushov, D. V.; Voth, G. A. J. Am. Chem. Soc. 2003, 125, 7470-7478.

(20) Paddon-Row, M. N. Adv. Phys. Org. Chem. 2003, 38, 1-85.

(21) Wallrapp, F.; Voityuk, A.; Guallar, V. J. Chem. Theory Comput. 2009, 5, 3312-3320.

(22) Wallrapp, F.; Voityuk, A.; Guallar, V. J. Chem. Theory Comput. 2010, 6, 3241-3248.

(23) Cordes, M.; Jacques, O.; Köttgen, A.; Jasper, C.; Boudebous, H.; Giese, B. Adv. Synth. Catal. 2008, 350, 1053-1062.

(24) Giese, B.; Napp, M.; Jacques, O.; Boudebous, H.; Taylor, A. M.; Wirz, J. Angew. Chem., Int. Ed. 2005, 44, 4073-4075.

(25) Meggers, E.; Michel-Beyerle, M. E.; Giese, B. J. Am. Chem. Soc. 1998, 120, 12950-12955.

(26) Meggers, E.; Kusch, D.; Spichty, M.; Wille, U.; Giese, B. Angew. Chem., Int. Ed. 1998, 37, 460-462.

(27) Isied, S. S.; Taube, H. J. Am. Chem. Soc. 1973, 95, 8198-8200.

(28) Isied, S. S. Prog. Inorg. Chem. 1984, 32, 443-517.

(29) Isied, S. S.; Vassilian, A. J. Am. Chem. Soc. 1984, 106, 17321736.

(30) Isied, S. S.; Vassilian, A.; Wishart, J. F.; Creutz, C.; Schwarz, H. A.; Sutin, N. J. Am. Chem. Soc. 1988, 110, 635-637.

(31) Isied, S. S.; Ogawa, M. Y.; Wishart, J. F. Chem. Rev. 1992, 92, 381-394.

(32) Ogawa, M. Y.; Moreira, I.; Wishart, J. F.; Isied, S. S. Chem. Phys. 1993, 176, 589-600.

(33) Isied, S. S.; Moreira, I.; Ogawa, M. Y.; Vassilian, A.; Arbo, B.; Sun, J. J. Photochem. Photobiol. A: Chem. 1994, 82, 203-210.

(34) Malak, R. A.; Gao, Z. N.; Wishart, J. F.; Isied, S. S. J. Am. Chem. Soc. 2004, 126, 13888-13889.

(35) Ungar, L. W.; Newton, M. D.; Voth, G. A. J. Phys. Chem. B 1999, 103, $7367-7382$. 
(36) Shin, Y. G. K.; Newton, M. D.; Isied, S. S. J. Am. Chem. Soc. 2003, 125, 3722-3732.

(37) Issa, J. B.; Krogh-Jespersen, K.; Isied, S. S. J. Phys. Chem. C 2010, 114, 20809-20812.

(38) Cordes, M.; Giese, B. Chem. Soc. Rev. 2009, 38, 892-901.

(39) Giese, B.; Wang, M.; Gao, J.; Stoltz, M.; Müller, P.; Graber, M. J. Org. Chem. 2009, 74, 3621-3625.

(40) Wang, M.; Gao, J.; Müller, P.; Giese, B. Angew. Chem., Int. Ed. 2009, 48, 4232-4234.

(41) Gao, J. A.; Müller, P.; Wang, M.; Eckhardt, S.; Lauz, M.; Fromm, K. M.; Giese, B. Angew. Chemie, Int. Ed. 2011, 50, 1926-1930.

(42) Gampp, H.; Maeder, M.; Meyer, C. J.; Zuberbühler, A. D. Talanta 1985, 32, 95-101.

(43) Gampp, H.; Maeder, M.; Meyer, C. J.; Zuberbühler, A. D. Talanta 1985, 32, 257-264.

(44) Kümin, M.; Sonntag, L.; Wennemers, H. J. Am. Chem. Soc. 2007, 129, 466-467.

(45) Long, Y.; Abu-Irhayem, E.; Kraatz, H. Chem.-Eur. J. 2005, 11, 5186-5194.

(46) Giese, B.; Graber, M.; Cordes, M. Curr. Op. Chem. Biol. 2008, $12,755-759$.

(47) Newton, M. D. Chem. Rev. 1991, 91, 767.

(48) Sheu, S.; Yang, D.; Selzle, H. L.; Schlag, E. W. J. Phys. Chem. A 2002, 106, 9390-9396.

(49) Marcus, R. A. J. Chem. Phys. 1956, 24, 966-978.

(50) Marcus, R. A. J. Chem. Phys. 1956, 24, 979-989.

(51) Marcus, R. A. Annu. Rev. Phys. Chem. 1964, 15, 155-196.

(52) Krapf, S.; Koslowski, T.; Steinbrecher, T. Phys. Chem. Chem. Phys. 2010, 12, 9516-9525.

(53) Woiczikowski, P. B.; Kubař, T.; Gutiérrez, R.; Caetano, R. A.; Cuniberti, G.; Elstner, M. J. Chem. Phys. 2009, 130, 215104.

(54) Woiczikowski, P. B.; Steinbrecher, T.; Kubar, T.; Elstner, M. J. Phys. Chem. B 2011, 115, 9846-9863.

(55) Kubar, T.; Woiczikowski, P. B.; Cuniberti, G.; Elstner, M. J. Phys. Chem. B 2008, 112, 7937-7947.

(56) Kubar, T.; Elstner, M. J. Phys. Chem. B 2008, 112, 8788-8798.

(57) van der Spoel, D.; Lindahl, E.; Hess, B.; Groenhof, G.; Mark, A.

E.; Berendsen, H. J. C. J. Comput. Chem. 2005, 26, 1701-1718.

(58) Hess, B.; Kutzner, C.; van der Spoel, D.; Lindahl, E. J. Chem. Theory Comput. 2008, 4, 435-447.

(59) Hess, B. J. Chem. Theory Comput. 2008, 4, 116-122.

(60) Humphrey, W.; Dalke, A.; Schulten, K. J. Mol. Graph. 1996, 14, $33-38$.

(61) Schaftenaar, G.; Noordik, J. H. J. Comput. Aided Mol. Design 2000, 14, 123-134.

(62) Hornak, V.; Abel, R.; Okur, A.; Strockbine, B.; Roitberg, A.; Simmerling, C. Proteins: Struct., Funct., Genet. 2006, 65, 712-725.

(63) Horn, H.; Swope, W.; Pitera, J.; Madura, J.; Dick, T.; Hura, G.; Head-Gordon, T. J. Chem. Phys. 2004, 120, 9665-9678.

(64) Horn, H.; Swope, W.; Pitera, J. J. Chem. Phys. 2005, 123, 194504.

(65) Bayly, C.; Cieplak, P.; Cornell, W.; Kollman, P. J. Phys. Chem. 1993, 97, 10269.

(66) Cornell, W.; Cieplak, P.; Bayly, C.; Kollman, P. J. Am. Chem. Soc. 1993, 115, 9620-9631.

(67) Wang, J.; Cieplak, P.; Kollman, P. J. Comput. Chem. 2000, 21, 1049-1074.

(68) Hush, N. S. Trans. Farad. Soc. 1961, 57, 557-580.

(69) Levich, V. G.; Dogonadze, R. R. Doklady Akademii Nauk SSSR 1959, 124, 123-126.

(70) Jortner, J. Biochim. Biophys. Acta 1980, 594, 193-230.

(71) Skourtis, S. S.; Balabin, I. A.; Kawatsu, T.; Beratan, D. N. Proc.

Natl. Acad. Sci. 2005, 102, 3552-3557.

(72) Jang, S.; Newton, M. D. J. Chem. Phys. 2005, 122, 024501.

(73) Troisi, A.; Nitzan, A.; Ratner, M. A. J. Chem. Phys. 2003, 119, 5782-5788.

(74) Berlin, Y. A.; Grozema, F. C.; Siebbeles, L. D. A.; Ratner, M. A. J. Phys. Chem. C 2008, 112, 10988-11000.

(75) Neria, E.; Nitzan, A. J. Chem. Phys. 1993, 99, 1109-1123.
(76) Lockwood, D. M.; Cheng, Y. K.; Rossky, P. J. Chem. Phys. Lett. 2001, 345, 159-165.

(77) Prezhdo, O. V.; Rossky, P. J. J. Chem. Phys. 1997, 107, 58635878 .

(78) Prezhdo, O. V.; Rossky, P. J. Phys. Rev. Lett. 1998, 81, 52945297.

(79) Onuchic, J. N.; Wolynes, P. G. J. Phys. Chem. 1988, 92, 64956503

(80) Bialek, W.; Bruno, W. J.; Joseph, J.; Onuchic, J. N. Photosynth. Res. 1989, 22, 15-27.

(81) Box, G. E. P.; Jenkins, G. Time Series Analysis: Forecasting and Control; Holden-Day: Oakland, CA, 1976.

(82) Troisi, A.; Ratner, M. A.; Zimmt, M. B. J. Am. Chem. Soc. 2004, 126, 2215-2224.

(83) Moser, C. C.; Keske, J. M.; Warncke, K.; Farid, R. S.; Dutton, P. L. Nature 1992, 355, 796-802.

(84) Hopfield, J. J. Proc. Natl. Acad. Sci. U.S.A. 1974, 71, 3640-3644.

(85) Skourtis, S. S.; Beratan, D. N. Adv. Chem. Phys. 1999, 106, 377452.

(86) Kurnikov, I. V.; Beratan, D. N. J. Chem. Phys. 1996, 105, 95619573 .

(87) Beratan, D. N.; Betts, J. N.; Onuchic, J. N. Science 1991, 252, 1285-1288.

(88) Jones, M. L.; Kurnikov, I. V.; Beratan, D. N. J. Phys. Chem. A 2002, 106, 2002-2006.

(89) Winkler, J. R.; Gray, H. B. J. Biol. Inorg. Chem. 1997, 2, 399404.

(90) Langen, R.; Colon, J. L.; Casimiro, D. R.; Karpishin, T. B.; Winkler, J. R.; Gray, H. B. J. Biol. Inorg. Chem. 1996, 3, 221-225.

(91) Skov, L. K.; Pascher, T.; Winkler, J. R.; Gray, H. B. J. Am. Chem. Soc. 1998, 120, 1102-1103.

(92) Regan, J. J.; DiBilio, A. J.; Langen, R.; Skov, L. K.; Winkler, J. R.; Gray, H. B. Chem. Biol. 1995, 2, 489-496.

(93) Gray, H. B.; Winkler, J. R. Pure Appl. Chem. 1992, 64, 12571262

(94) Blumberger, J.; Lamoureux, G. Mol. Phys. 2008, 106, 15971611 .

(95) Sulpizi, M.; Raugei, S.; VandeVondele, J.; Carloni, P.; Sprik, M. J. Phys. Chem. B 2007, 111, 3969-3976.

(96) Marchi, M.; Gehlen, J. N.; Chandler, D.; Newton, M. J. Am. Chem. Soc. 1993, 115, 4178-4190.

(97) Kuharski, R. A.; Bader, J. S.; Chandler, D.; Sprik, M.; Klein, M. L.; Impey, R. W. J. Chem. Phys. 1988, 89, 3248-3257.

(98) Ando, K. J. Chem. Phys. 2001, 114, 9470-9477.

(99) Ceccarelli, M.; Marchi, M. J. Phys. Chem. B 2003, 107, 56305641 .

(100) Algen, R. G.; Parson, W. W.; Chu, C. T.; Warshel, A. J. Am. Chem. Soc. 1995, 117, 12284-12298.

(101) Schulten, K.; Tesch, M. Chem. Phys. 1991, 158, 421-446.

(102) King, G.; Warshel, A. J. Chem. Phys. 1990, 93, 8682-8692.

(103) Malagoli, M.; Bredas, J. L. Chem. Phys. Lett. 2000, 327, 13-17.

(104) Berlin, Y. A.; Hutchison, G. R.; Rempala, P.; Ratner, M. A.; Michl, J. J. Phys. Chem. A 2003, 107, 3970-3980.

(105) Sakanoue, K.; Motoda, M.; Sugimoto, M.; Sakaki, S. J. Phys. Chem. A 1999, 103, 5551-5556.

(106) Klimkans, A.; Larsson, S. Int. J. Quantum Chem. 2000, 77, 211-220.

(107) Olofsson, J.; Larsson, S. J. Phys. Chem. B 2001, 105, 1039810406.

(108) Klimkans, A.; Larsson, S. Chem. Phys. 1994, 189, 25-31.

(109) Lemaur, V.; da Silva Filho, D. A.; Coropceanu, V.; Lehmann, M.; Geerts, Y.; Piris, J.; Debije, M. G.; van de Craats, A. M.; Senthilkumar, K.; Siebbeles, L. D.; Warman, J. M.; Bredas, J.; Cornil, J. J. Am. Chem. Soc. 2004, 126, 3271-3279.

(110) Hohenberg, P.; Kohn, W. Phys. Rev. B 1964, 136, 864-871.

(111) Kohn, W.; Sham, L. J. Phys. Rev. A 1965, 140, 1133-1138.

(112) Becke, A. D. Phys. Rev. A 1988, 38, 3098-3100.

(113) Becke, A. D. J. Chem. Phys. 1993, 98, 5648-5652. 
(114) Hariharan, P. C.; Pople, J. A. Theor. Chim. Acta 1973, 28, $213-$ 222.

(115) Hehre, W. J.; Ditchfield, R.; Pople, J. A. J. Chem. Phys. 1972, 56, 2257-2261.

(116) Amini, A.; Harriman, A. J. Photochem. Photobiol. C 2003, 4, 155-177.

(117) Voityuk, A. A. J. Chem. Phys. 2005, 123, 034903.

(118) Senthilkumar, K.; Grozema, F. C.; Guerra, C. F.; Bickelhaupt, F. M.; Lewis, F. D.; Berlin, Y. A.; Ratner, M. A.; Siebbeles, L. D. A. J. Am. Chem. Soc. 2005, 127, 14894-14903.

(119) Porezag, D.; Frauenheim, T.; Kohler, T.; Seifert, G.; Kaschner, R. Phys. Rev. B 1995, 51, 12947-12957.

(120) Elstner, M.; Porezag, D.; Jungnickel, G.; Elsner, J.; Haugk, M.; Frauenheim, T.; Suhai, S.; Seifert, G. Phys. Rev. B 1998, 58, 72607268.

(121) Gutierrez, R; Caetano, R.; Woiczikowski, P. B.; Kubař, T.; Elstner, M.; Cuniberti, G. New J. Phys. 2010, 12, 023022.

(122) Kubar, T.; Kleinekathöfer, U.; Elstner, M. J. Phys. Chem. B 2009, 113, 13107-13117.

(123) Beratan, D. N.; Onuchic, J. N.; Winkler, J. R.; Gray, H. B. Science 1992, 258, 1740-1741.

(124) de la Lande, A.; Salahub, D. R. J. Mol. Struct. THEOCHEM 2010, 943, 115-120.

(125) Ayala, R.; Sprik, M. J. Chem. Theory Comput. 2006, 2, 14031415.

(126) VandeVondele, J.; Ayala, R.; Sulpizi, M.; Sprik, M. J. Electroanalyt. Chem. 2007, 607, 113-120.

(127) Blumberger, J.; Sprik, M. J. Phys. Chem. B 2005, 109, 67936804.

(128) Blumberger, J.; Sprik, M. Theor. Chem. Acc. 2006, 115, 113126.

(129) Tateyama, Y.; Blumberger, J.; Sprik, M.; Tavernelli, I. J. Chem. Phys. 2005, 122, 234505.

(130) Blumberger, J.; Tavernelli, I.; Klein, M. L.; Sprik, M. J. Chem. Phys. 2006, 124, 064507.

(131) Liu, H. Y.; Zhang, Y. K.; Yang, W. J. Am. Chem. Soc. 2000, 122, 6560-6570.

(132) Hasegawa, J. Y.; Ishida, M.; Nakatsuji, H.; Lu, Z. Y.; Liu, H. Y.; Yang, W. J. Phys. Chem. B 2003, 107, 838-847.

(133) Zeng, X.; Hu, H.; Hu, X.; Cohen, A. J.; Yang, W. J. Chem. Phys. 2008, 128, 124510.

(134) Ayala, R.; Sprik, M. J. Phys. Chem. B 2008, 112, 257-269.

(135) Campbella, S.; Beauchamp, J. L.; Rempe, M.; Lichtenberger, D. L. Int. J. Mass. Spec. Ion Proc. 1992, 117, 83-99.

(136) Kishor, S.; Dhayal, S.; Mathur, M.; Ramaniah, L. M. Mol. Phys. 2008, 106, 2289-2300.

(137) Dehareng, D.; Dive, G. Int. J. Mol. Sci. 2004, 5, 301-332.

(138) Kubař, T.; Elstner, M. J. Phys. Chem. B 2009, 113, 5653-5656.

(139) Steinbrecher, T.; Joung, I; Case, D. A. J. Comput. Chem. 2011, $32,3253-3263$.

(140) Steinbrecher, T.; Koslowski, T.; Case, D. A. J. Phys. Chem. B 2008, 112, 16935-16944.

(141) Kubař, T.; Elstner, M. J. Phys. Chem. B 2010, 114, 1122111240.

(142) de la Lande, A.; Babcock, N. S.; Rezac, J.; Sanders, B. C.; Salahub, D. R. Proc. Natl. Acad. Sci. U.S.A. 2010, 107, 11799-11804. (143) Lin, J.; Balabin, I. A.; Beratan, D. N. Science 2005, 310, 13111313. 